\title{
Analysis of status and waiting period of heart and lung transplant recipients in single center
}

\author{
In Ok Kim
}

Organ Transplantation Center, Asan Medical Center, University of Ulsan College of Medicine, Seoul, Korea

Background: Heart and lung transplantation is not only a lifesaving method for end-stage heart and lung failure patients, but also a treatment method that improves the quality of life. As the survival rate of heart and lung transplantation improved, the number gradually increased, and by $2020,1,887$ heart transplants and 878 lung transplants were performed in Korea. In particular, as the number of transplants has increased rapidly since 2010, 173 heart transplants and 150 lung transplants were performed only in 2020. In the end of 2020, the number of heart and lung transplantation waiting list registered in Korean Network for Organ Sharing (KONOS) is continuously increasing to 774 and 323 respectively. In the case of heart and lung transplantation that is dependent on a deceased donor, the management of the waiting patient for transplantation is also becoming more important.

Methods: Since the establishment of KONOS in February 2000, the latest standard of transplant status level for heart and lung transplant recipients was revised in July of 2017 and August of 2018. By the end of June 2021, our center performed 815 heart and 179 lung transplants, after the first heart transplantation on November 11, 1992 and lung transplantation on October 29, 2008. In this study, the status level and waiting period of heart and lung transplant recipients were retrospectively investigated through KONOS network data and medical records.

Results: The sex of transplant recipients was 565 male (70\%), 250 female (30\%) with heart, 115 male (65\%) and 64 female (35\%) with lung. The average age at the time of transplantation was 45 years (range, 3 months -74 years) for heart and 47.6 years (range, 14 months -74 years) for lung. At the time of heart transplantation, the final status (S) and mean waiting period were S0(153 patients, 129 days), S1 (321 patients, 202 days), S2 (166 patients, 116 days), S3 (97 patients, 194 days), lungs were S0 (120 patients, 113 days), S1 (47 patients, 353 days), S2 (7 patients, 335 days), and S3 (3 patients, 91 days). After the status level was raised, the waiting period was heart/lung S0 (20/24 days), S1 (60/200 days), and S2 (72/292 days). The average waiting period of transplants nationwide compared to the hospital by year was 210/214 days (2016), 219/234 days (2017), 246/228 days (2018), $226 / 211$ days (2019), lung was 112/116 days (2016), 107/116 days (2017), 167/147 days (2018), and 317/234 days (2019).

Conclusions: As the waiting period become longer, it is important to provide practical information about the average waiting time to heart and lung transplant patients and the maintenance of optimal health during the waiting period, and to care for the importance of rehabilitation. Due to the shortage of deceased-dead donors, efforts are being made to increase transplant surgery, such as expanded category deceased-donors or new techniques for living donor lung lobar transplantation. It can be used for basic data for transplant waiting management and education. It is necessary to supplement the Status Scoring System Standards comparing additional studies and advanced systems.

Corresponding author: In Ok Kim

E-mail:yinoky@hanmail.net

(c) The Korean Society for Transplantation

This is an Open Access article distributed under the terms of the Creative Commons Attribution Non-Commercial License (http://creativecommons.org/licenses/by-nc/4.0/) which permits unrestricted non-commercial use, distribution, and reproduction in any medium, provided the original work is properly cited. 